Jacques Piette

Patricia DefechereuxThibaut de Maisières

Laurence

Baudoux-Tebache

Catherine

Sadzot-Delvaux

Bernard Rentier

\title{
La régulation des cycles infectieux du virus de la varicelle et du zona
}

Le virus de la varicelle et du zona (VZV) est responsable de ces deux syndromes cliniques distincts. Pendant la période d'incubation de la varicelle, le virus se multiplie au niveau de divers organes. Il peut ensuite gagner le système nerveux périphérique et y rester latent pendant une période variable, avant d'être réactivé sous la forme du zona. Trois protéines virales ont été mises en cause dans cette réactivation du virus : la phosphoprotéine nucléaire virale IE62, synthétisée durant la phase précoce immédiate (IE) du cycle et qui active l'expression de toutes les classes de gènes viraux; la protéine IE4, principalement cytoplasmique, qui agit en synergie avec IE62 pour activer des promoteurs des gènes du VZV; la phosphoprotéine IE63, seule à être abondamment synthétisée pendant la latence dans les neurones des ganglions sensoriels, qui semble constituer une cible pour le système immunitaire. Sa présence lors d'une infection productive mais également pendant la latence est un cas unique parmi les Alphaherpesvirus et suggère qu'elle pourrait jouer un rôle dans l'établissement et le contrôle de la latence.

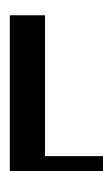

e virus de la varicelle et du zona présente la caractéristique inhabituelle d'être directement responsable de deux maladies communes et distinctes, la varicelle et le zona. C'est T.H. Weller [1] qui le premier isola le virus, puis démontra l'identité entre les virus responsables de ces deux affections, ce qui l'amena à proposer le nom de Varicella-Zoster Virus (VZV). Plus de cent vingt Herpesvirus ont pu être identifiés à l'heure actuelle et parmi ceux-ci, huit peuvent infecter l'homme (le virus herpes simplex de type 1 , HSV-1 ; le virus herpes simplex de type 2, HSV-2; le virus de la varicelle et du zona; le cytomégalovirus CMV-; le virus d'Epstein-Barr, EBV; les virus HHV-6, HHV-7 et $\mathrm{HHV}-8$, pour human Herpesvirus 6,7 et $8(\mathrm{~m} / \mathrm{s}$ $n^{\circ} 11$, vol. 11, p. 1605). Des critères biologiques ont permis de subdiviser cette énorme famille en trois sousclasses, les Alpha-, les Bêta- et les Gamma-herpesviridae. Le VZV, comme les virus Herpes simplex de type 1 et de type 2 (HSV-1 et HSV-2), appartient à la famille des Alphaherpesviridae, caractérisée par le neurotropisme, un cycle réplicatif rapide et fortement lytique, une gamme 
d'hôtes restreinte ainsi qu'une capacité d'entrer en latence au niveau du système nerveux périphérique.

Le VZV est un virus enveloppé d'un diamètre d'environ $200 \mathrm{~nm}$ (figure 1) [2]. Il comprend un ADN en double brin linéaire comportant 124884 paires de bases [3], protégé par une capside protéique icosaédrique composée de 162 capsomères hexagonaux. Un tégument protéique sépare la capside de l'enveloppe virale. Les protéines qui le composent, outre le rôle structural qu'elles jouent dans le virion, peuvent intervenir de façon prépondérante dans les étapes précoces de l'infection. L'enveloppe virale, acquise au sein de la cellule, est hérissée de projections glycoprotéiques.

\section{Les maladies}

La première infection par le VZV déclenche la varicelle, une maladie très contagieuse, mais généralement bénigne, survenant durant l'enfance [4]. Près de $100 \%$ de la population a un jour été en contact avec le virus qui peut être transmis par contact direct ou par voie aérienne. Le virus pénètre par les voies respiratoires et infecte la muqueuse respiratoire ainsi que les cellules des ganglions lymphatiques proches. Après quatre à cinq jours, le virus est disséminé une première fois par voie sanguine dans tout l'organisme, lui permettant d'infecter les cellules du système réticulo-endothélial dans lesquelles il se multiplie. Alors survient une seconde

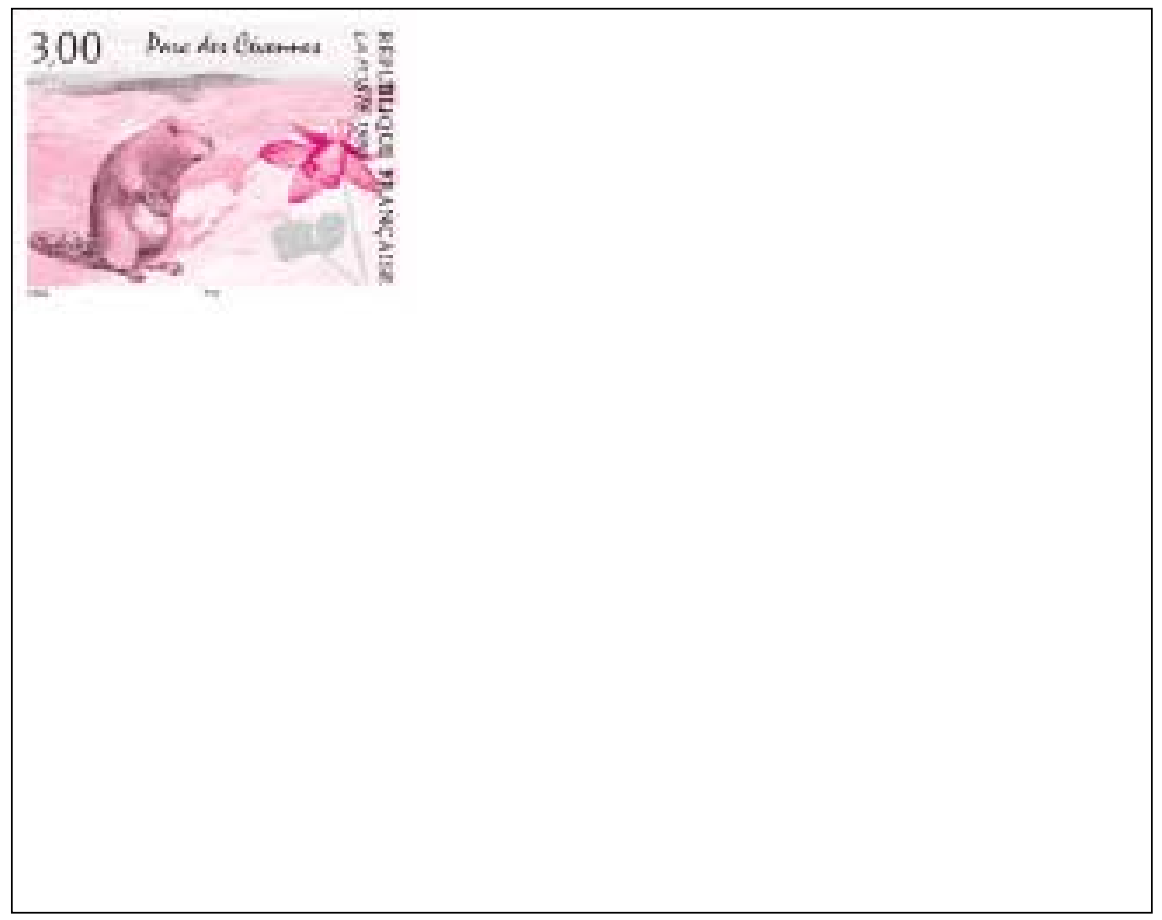

Figure 1. Schéma de la structure du VZV. La particule virale a un diamètre d'environ $200 \mathrm{~nm}$ et est entourée d'une enveloppe lipidique (1) dans laquelle sont ancrées plusieurs glycoprotéines (2) appelées $g E, g B, g C, g H$, et gl. Sous l'enveloppe virale, se trouve un tégument protéique (3) qui renferme plusieurs protéines régulatrices comme, par exemple, IE4, IE62 et IE63. La capside du VZV (4) est icosahédrique et composée de 162 capsomères hexagonaux. À l'intérieur de la capside est enfermé l'ADN en double chaîne qui comprend 124884 paires de base. L'ADN est composé de deux segments uniques: UL (104 836 bp) et Us (5 232 bp). Ceux-ci sont entourés d'éléments répétés: $T R^{L}$ et $I R^{L}(88,5 \mathrm{pb})$ et $T R^{S}$ et $I R^{S}(7319,5 \mathrm{pb})$. L'ADN viral comprend 71 cadres de lecture ouverts dont trois sont en deux copies (ORF 62, 63 et 64). En encart, photographie en microscopie électronique d'une particule virale (grossissement $\times 100000$ ). dissémination par voie sanguine au cours de laquelle le virus gagne les cellules épithéliales cutanées provoquant l'apparition des lésions vésiculaires caractéristiques. Celle-ci prend fin après trois jours, grâce à l'intervention du système immunitaire de l'hôte. Si l'immunité se révèle déficiente, la dissémination du pathogène n'est pas arrêtée et il peut alors atteindre les poumons, le foie et d'autres organes internes et représenter ainsi une menace sérieuse pour l'organisme. Il faut noter que, malgré un aspect anodin, la varicelle entraîne des complications d'ordre neurologique (encéphalites, méningites...) chez un à quatre patients sur mille [4].

Au cours de cette première infection, le virus peut gagner les ganglions du système nerveux périphérique, soit par la voie sanguine, soit en pénétrant par l'extrémité de nerfs sensoriels innervant la zone cutanée infectée et en se déplaçant le long de l'axone jusqu'au ganglion. C'est au sein de ces ganglions sensoriels que le virus entre dans une phase d'infection latente qui peut se prolonger pendant de nombreuses années. Aucun effet cytopathique ne semble associé à l'établissement de la latence et on a pu déterminer si un processus de réplication du virus dans les ganglions était nécessaire [2].

La réactivation du virus resté latent est responsable du zona [4]. Cette seconde affection est caractérisée par des douleurs aiguës et l'apparition de vésicules cutanées dans une région généralement limitée au territoire cutané innervé par un seul nerf rachidien. La limitation de la zone de réactivation reflète bien l'implication d'un seul ganglion, indiquant que le virus se réactive au niveau de celui-ci puis migre le long des axones pour infecter les cellules épithéliales. Si n'importe quel ganglion sensoriel peut être impliqué dans une réactivation, c'est cependant au niveau des ganglions thoraciques et lombaires qu'elle est le plus fréquemment observée («ceinture de feu»).

Souvent rencontrée chez les personnes âgées, la réactivation du virus semble liée à un affaiblissement de la réponse immunitaire et, plus particulièrement, de l'immunité cellulaire. On estime qu'une personne sur deux atteignant l'âge de 80 ans développe 
ou a développé un zona. Comme pour la varicelle, la réactivation $\mathrm{du}$ virus chez les patients immunodéprimés peut s'accompagner de lésions plus importantes, voire d'une dissémination (zona généralisé) vers des organes internes, pouvant provoquer des pneumonies, des hépatites ou des encéphalites. Ainsi, le VZV est un des pathogènes viraux majeurs réactivés chez les malades du SIDA.

\section{Réplication du virus et latence}

En culture de cellules, le VZV reste fortement associé aux cellules qu'il infecte. De plus, les rares virions libérés dans le milieu extracellulaire s'avèrent très instables, ce qui rend problématique l'étude de leurs propriétés biologiques, notamment en raison de la difficulté rencontrée pour construire des virus mutants [2]. Malgré ces obstacles importants qui ont freiné la recherche sur les mécanismes de réplication, de latence et de réactivation, des informations précieuses ont été acquises ces dernières années grâce à l'avènement de la biologie moléculaire, la mise au point de modèles animaux d'infection latente et la caractérisation approfondie de virus appartenant à la même famille. Toutefois, la simple extrapolation des observations faites sur le HSV-1, prototype des Alphaherpesvirus, se révèle souvent trompeuse.

Le cycle réplicatif du VZV (figure 2) débute par son adsorption sur les cellules cibles par l'intermédiaire de récepteurs spécifiques encore indéterminés, bien que les récepteurs du mannose-6-phosphate et du sulfate d'héparine semblent jouer un rôle important. La capside et les protéines du tégument pénètrent dans la cellule et migrent dans le cytoplasme. L'ADN viral (accompagné probablement de protéines du tégument) gagne le noyau dans lequel il est décapsidé et où les gènes viraux sont transcrits séquentiellement. Les gènes précoces immédiats (immediate-early, $I E$ ou $\alpha$ ) sont exprimés les premiers en l'absence de synthèse protéique $d e$ novo. Puis, les gènes précoces (early, $E$ ou $\beta$ ) sont transcrits, avant la réplication de l'ADN du virus. Ces deux classes de gènes codent pour des protéines impliquées, respectivement,

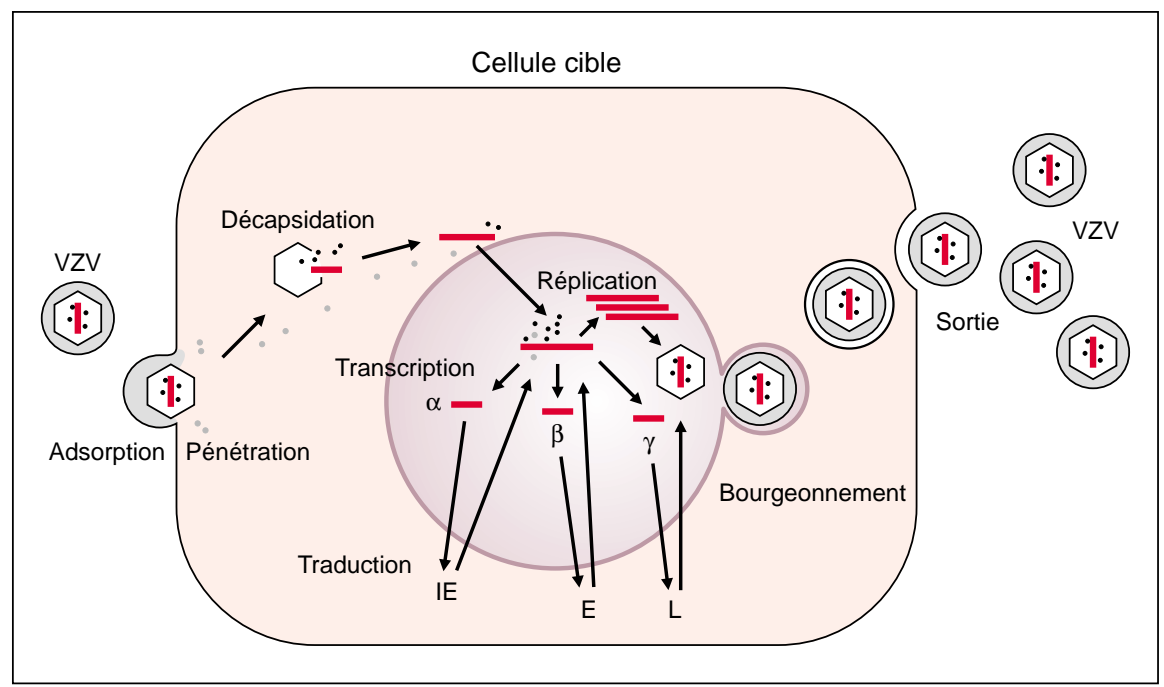

Figure 2. Cycle infectieux du VZV. Le cycle réplicatif du VZV comprend de nombreuses phases successives: l'adsorption de la particule sur la membrane de la cellule cible par interaction entre certaines glycoprotéines du virus et les récepteurs du mannose 6-phosphate ou du sulfate d'héparine, pénétration de la capside et du tégument, décapsidation et migration de I'ADN dans le noyau; transcription séquentielle des trois classes de gènes $(\alpha$, $\beta$ et $\gamma)$, traduction des protéines virales (IE, $E$ et $L$ ), et réplication de I'ADN viral. La dernière étape comprend l'assemblage de la capside et l'incorporation de l'ADN viral avec acquisition de l'enveloppe par bourgeonnement à la membrane nucléaire.

dans la régulation de l'expression des gènes et la réplication de l'ADN viral. L'expression des gènes tardifs (late, $L$ ou $\gamma)$ a lieu ensuite, permettant la production des protéines virales structurales. La dernière étape du cycle consiste en l'assemblage des virions : les protéines de structure synthétisées dans le cytoplasme migrent vers le noyau et s'assemblent en capsides tout en incorporant un génome viral. Les processus par lesquels le virus acquiert son enveloppe et quitte la cellule sont encore sujets à controverse.

Bien que partageant une même ultrastructure morphologique (figure 1) et un même schéma de cycle réplicatif, la latence moléculaire du VZV se distingue de celle des autres Alphaherpesvirus. Dans le cas du HSV-1, du Pseudorabies (PRV) responsable de la maladie d'Aujesky chez le porc ou du BHV-1 responsable de la rhinotrachéite infectieuse bovine, il est clairement établi que les neurones sont le siège de l'infection latente et qu'une seule région du génome viral est transcriptionnellement active dans ces cellules. Les transcrits résultant de cette activité, appelés LAT (latency associated transcripts), sont antiparal- lèles à l'ARN messager d'un gène $I E$ important [5]. Jusqu'à présent, aucune protéine correspondant à ces transcrits caractéristiques de la phase latente n'a pu être mise en évidence, et les fonctions exactes des LAT ne sont pas encore établies. Bien qu'ils ne semblent pas jouer un rôle majeur dans l'établissement et le maintien de la latence, les LAT pourraient intervenir lors de la réactivation. En revanche, dans le cas du VZV, la nature des cellules contenant le génome viral est encore sujette à controverse puisque, selon les auteurs, les neurones et/ou les cellules satellites peuvent être le siège de l'infection latente [6-8]. Par ailleurs, pendant la latence du VZV, plusieurs transcrits de gènes exprimés également au cours du cycle lytique peuvent être détectés [6-8]. Il s'agit des ARN messagers des gènes 4, 21, 29, 62 et 63. Parmi ceux-ci, trois codent pour des protéines régulatrices IE (4, 62 et 63). Ces différentes caractéristiques moléculaires qui pourraient refléter les particularités biologiques du VZV sont résumées dans le Tableau I.

Malgré de nombreuses études, les mécanismes impliqués dans la 
Tableau I

DIFFÉRENCES BIOLOGIOUES ENTRE LE HSV-1 ET LE VZV

\begin{tabular}{|c|c|c|}
\hline Caractéristiques & VZV & HSV-1 \\
\hline \multicolumn{3}{|l|}{ Réactivation } \\
\hline Fréquence de récurrences & $\begin{array}{l}\text { généralement, une } \\
\text { augmente avec l'âge }\end{array}$ & $\begin{array}{l}\text { de quelques-unes à plusieurs centaines } \\
\text { diminue avec le temps }\end{array}$ \\
\hline $\begin{array}{l}\text { Séropositifs avec des récurrences } \\
\text { symptomatiques }\end{array}$ & $10-20 \%$ de séropositifs & $20-47 \%$ de séropositifs \\
\hline Distribution & dermatome & lésion focale \\
\hline Sites de latence & $\begin{array}{l}\text { cellules neuronales ou cellules } \\
\text { satellites } \\
\text { ganglion(s) sensoriel(s) }\end{array}$ & $\begin{array}{l}\text { neurones } \\
\text { ganglion sacré/trijumeau }\end{array}$ \\
\hline Douleur & $\begin{array}{l}\text { sévère } \\
\text { (névralgies postherpétiques) }\end{array}$ & moyenne \\
\hline $\begin{array}{l}\text { Réactivation par les UV } \\
\text { Dispersion asymptomatique } \\
\text { Délai de réactivation } \\
\text { après greffe de moelle }\end{array}$ & $\begin{array}{l}\text { non } \\
\text { non } \\
2-6 \text { mois }\end{array}$ & $\begin{array}{l}\text { oui } \\
\text { oui } \\
1-4 \text { semaines }\end{array}$ \\
\hline \multicolumn{3}{|l|}{ Biologie } \\
\hline Modèles animaux & $\begin{array}{l}\text { peu } \\
\text { reproduisent difficilement } \\
\text { l'infection chez I'homme }\end{array}$ & $\begin{array}{l}\text { plusieurs } \\
\text { reproduisent I'infection observée } \\
\text { chez l'homme }\end{array}$ \\
\hline $\begin{array}{l}\text { Expression génique pendant } \\
\text { la latence }\end{array}$ & transcription de gènes $I E$ et $E$ & $\begin{array}{l}\text { présence de } L A T \text { (transcrits associés } \\
\text { à la latence) }\end{array}$ \\
\hline $\begin{array}{l}\text { Synthèse protéique pendant } \\
\text { la latence }\end{array}$ & oui & non \\
\hline $\begin{array}{l}\text { Comportement en culture } \\
\text { de cellules }\end{array}$ & fortement associé aux cellules & présence dans le surnageant de culture \\
\hline
\end{tabular}

latence sont encore mal définis. Celle-ci apparaît cependant comme le résultat d'un équilibre délicat entre des facteurs viraux, cellulaires et immunitaires. Dans le cas du VZV, la présence de transcrits spécifiques témoigne de l'importance potentielle de ces séquences et des protéines pour lesquelles elles codent dans les événements moléculaires liés à l'infection latente.

\section{Régulation de l'expression des gènes viraux}

Chez les eucaryotes, les gènes codant pour des protéines sont transcrits par l'ARN polymérase II et toutes les étapes de l'expression d'un gène de classe II, depuis le début de sa trans- cription jusqu'à la dégradation de la protéine mûre, peuvent être la cible d'une régulation étroite. La régulation de l'expression des 71 gènes du VZV est essentielle au bon déroulement de l'infection. Elle doit se révéler suffisamment précise pour contrôler l'expression en cascade des trois classes de gènes lors d'une infection productive, et suffisamment souple pour permettre, selon les conditions, le déclenchement, soit d'un cycle lytique, soit d'un cycle latent. Si la régulation de l'expression des gènes viraux s'exerce à différents niveaux de l'infection, le contrôle des premières étapes est toutefois primordial pour le déclenchement d'une infection lytique ou latente. Les protéines virales susceptibles d'intervenir préférentielle- ment dans la régulation de ces étapes initiales doivent, soit être apportées par le virus, soit être synthétisées durant les phases très précoces du cycle (protéines IE). Ainsi, quatre protéines régulatrices du VZV, à savoir les protéines codées par les gènes 4, 10, 62 et 63 ont été recensées [9-11].

Le produit du gène $10(\mathrm{ORF} 10 \mathrm{p})$ est une protéine tégumentaire [9], composée de 410 acides aminés qui présente une considérable similitude de séquence avec la protéine transactivatrice VP16 du HSV-1 impliquée dans le début de la transcription de ses gènes IE. Pourtant, l'ORF10p n'est pas indispensable à la réplication du VZV puisqu'un virus mutant, dont le gène 10 a été délété, se réplique comme la souche parentale [12]. Par 
ailleurs, l'ORF10p ne peut transactiver que le promoteur du gène IE62 mais pas ceux des autres gènes $I E$ du VZV [10]. Par conséquent, le déclenchement de la transcription des gènes $I E$ lors du cycle réplicatif du VZV semble différent de celui que l'on a rencontré chez le HSV-1 et impliquerait une protéine tégumentaire différente.

Par ailleurs, comme nous l'avons mentionné plus haut, les ARN messagers des gènes 4,62 et 63 ont été mis en évidence dans des cellules infectées de manière latente. Par conséquent, ces protéines focalisent l'attention dans le domaine de la régulation de l'expression des gènes.

\section{La protéine IE62, une protéine régulatrice majeure du VZV}

La phosphoprotéine IE62 (175 kDa) appartient à une famille de protéines régulatrices dont font partie des protéines IE de différents Alphaherpesvirus tels que l'ICP4 du HSV-1, l'IE180 du PRV et l'IE-1 de l'EHV-1 (equine herpesvirus de type 1). En effet, une importante conservation de la séquence en acides aminés entre ces diverses protéines a été observée [2, $3]$, ce qui a permis de les diviser en cinq régions intramoléculaires: les régions 2 et 4 très conservées sont séparées par les régions 1,3 et 5 présentant peu de similitudes (figure 3 ). Or, la protéine ICP4 joue un rôle essentiel dans les cycles infectieux du HSV-1 : des virus privés du gène codant pour cette protéine et inoculés à des souris ne sont capables ni de se répliquer au site d'inoculation ou dans les ganglions trijumeaux, ni d'établir une infection latente réactivable [13]. L'analogie fonctionnelle entre l'IE62 et l'ICP4 a été démontrée par des expériences de complémentation dans lesquelles l'IE62 restaure la viabilité de ces virus mutants en culture de cellules [14]. Ces études ont donc suggéré que l'IE62 pourrait également jouer un rôle régulateur crucial chez le VZV.

Cette hypothèse a été renforcée par des expériences d'expression transitoire dans lesquelles l'IE62 se montre capable de transactiver toutes les classes de gènes du virus (IE, $\mathrm{E}$ et $\mathrm{L}$ ) et d'augmenter l'infectivité de l'ADN
17]. En outre, sa concentration dans la cellule pourrait influencer ses activités car elle transactive l'expression de tous les gènes de manière dépendante de la dose [16] et est capable de régler positivement et négativement l'activité de son propre promoteur en fonction de sa concentration intracellulaire.

Les protéines IE62 et ORF10p sont toutes deux présentes dans le tégument du virion [9] mais, comme nous l'avons déjà mentionné, l'ORF10p est incapable de stimuler tous les promoteurs de gènes IE du VZV. Par conséquent, l'incorporation de l'IE62 dans le virion pourrait être un mécanisme compensatoire permettant d'assurer une expression rapide des gènes $I E$ après l'entrée du virus dans la cellule cible. Comme l'IE62 transactive l'expression de tous les gènes $I E$ et $E$ à faible concentration intracellulaire et réprime sa propre expression à plus forte concentration, on peut imaginer le rôle que pourrait jouer cette protéine au cours d'une infection productive du VZV: la protéine IE62 serait apportée, en faible concentration, dans le noyau de la cellule par le tégument du virus. Elle y déclencherait la phase IE en transactivant l'expression des gènes $I E$. Dans une deuxième étape, la protéine IE62 participerait à l'activation des promoteurs des gènes $E$, puis, seule ou en collaboration avec d'autres protéines virales, elle stimulerait l'expression des gènes $L$. Sa concentration intra-

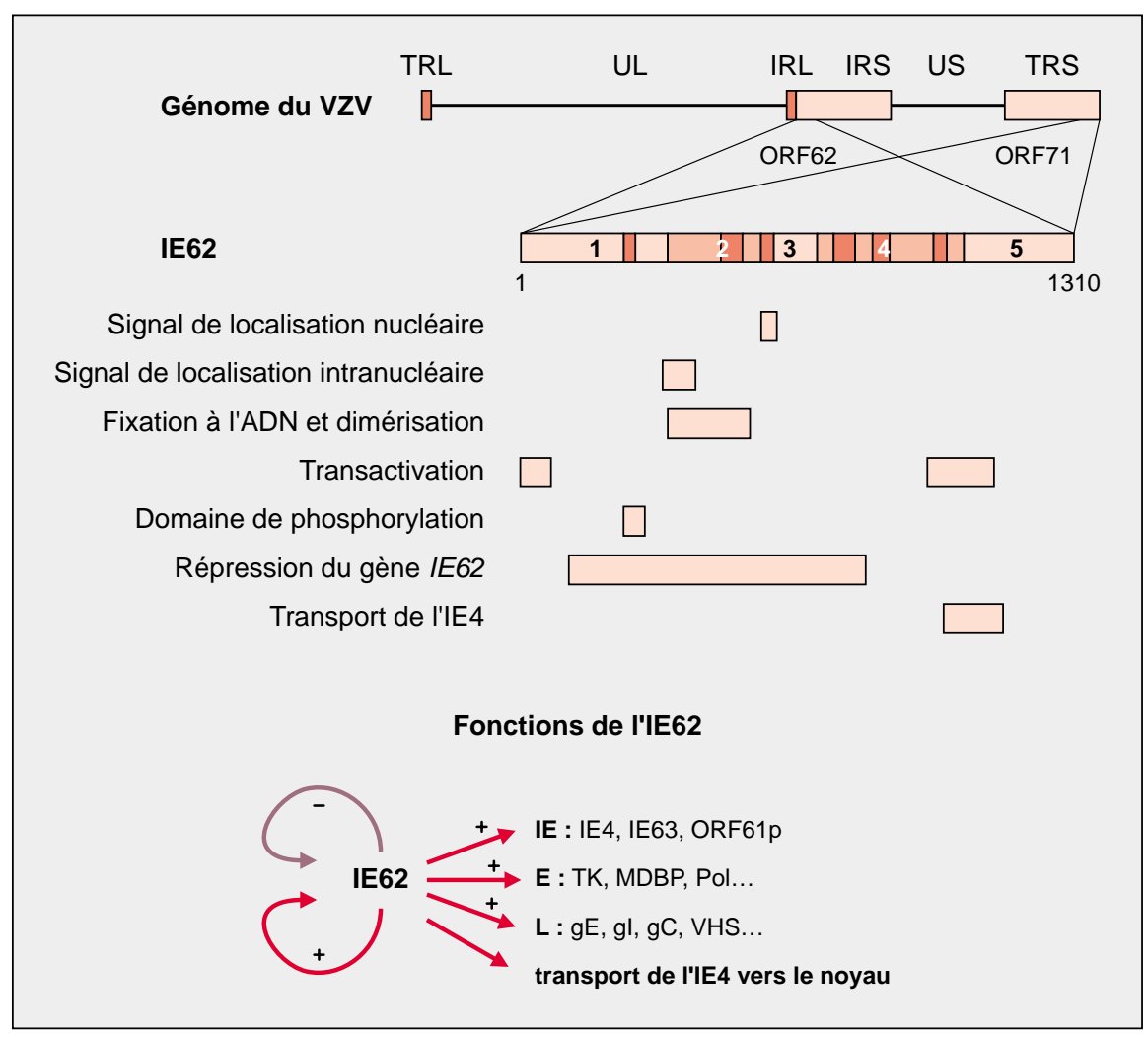

Figure 3. Anatomie de la protéine IE62 du VZV. Les ORF 62 et 71 du VZV codent pour une protéine de 1310 résidus appelée IE62 (175 kDa). Celle-ci présente de fortes similitudes avec la protéine ICP4 du HSV-1 et la comparaison des séquences en acides aminés de ces deux protéines a permis de les diviser en cinq régions intramoléculaires: les régions 2 et 4, très conservées, sont séparées par les régions 1, 3 et 5 peu semblables. L'IE62 est un activateur transcriptionnel puissant capable de transactiver tous les promoteurs de gènes codant pour les protéines IE, E et $L$ du VZV et de contrôler son propre promoteur. Elle contient un domaine de fixation à I'ADN, deux domaines activateurs, un signal de localisation nucléaire et intranucléaire, un site de phosphorylation. De plus, IE62 possède la propriété de transférer la protéine IE4 du cytoplasme vers le noyau. 
cellulaire augmentant grâce à sa propre stimulation et aux activités d'autres protéines régulatrices [10, $15,18]$, les sites fixant la protéine IE62 pour stimuler l'expression du promoteur seraient saturés et elle réprimerait sa propre expression en se fixant sur d'autres sites de son promoteur.

L'IE62 a été définie comme un activateur transcriptionnel car la stimulation du promoteur du gène de la thymidine-kinase du VZV est directement corrélée à une augmentation de la concentration de son ARNm [2]. Or, tout activateur transcriptionnel possède, en général, plusieurs domaines fonctionnels: un domaine de fixation à l'ADN, un domaine activateur et un signal de localisation nucléaire (figure 3). L'ensemble de la région 2 de l'IE62, et plus particulièrement les acides aminés 472 à 633, possède des propriétés de fixation à l'ADN et s'y fixe sous forme de dimères [19]. Une séquence, identifiée à l'intérieur du domaine de fixation à l'ADN présente des similitudes frappantes avec l'hélice de l'homéodomaine reconnaissant l'ADN. Pourtant, l'IE62 présente peu de spécificité puisque dans des expériences d'empreinte à la DNase, la région 2 de l'IE62 masque plusieurs séquences le long des régions promotrices-régulatrices des gènes $4,17,28,29$ et 62 [20, et résultats non publiés]. Des études de mutagenèse couplées à des expériences d'expression transitoire ont permis d'identifier les domaines fonctionnels de l'IE62 [16]. Ainsi, les régions 2 et 4 , dont les séquences sont voisines dans l'IE62 et l'ICP4, correspondent aux entités fonctionnelles les plus importantes. Par ailleurs, les domaines nécessaires aux propriétés transactivatrices et à la propriété autorépressive ne sont pas identiques. En effet, la région 2, contenant le domaine de fixation à l'ADN, est indispensable aux propriétés régulatrices étudiées, tandis que la région 4 semble plus importante pour les activités stimulatrices que pour l'activité répressive de la protéine IE62. Une séquence de la région 4 nécessaire aux activités transactivatrices est riche en proline et en acides aminés hydrophobes. En mesurant les propriétés transactivatrices de protéines de fusion compo- sées de séquences de la protéine IE62 liées au domaine fixant l'ADN de la protéine GAL4, il est apparu qu'un autre domaine activateur riche en résidus acides était localisé dans la région 1, amino-terminale [21]. Il est intéressant de noter qu'un site de phosphorylation potentiel, composé d'une suite de sérines puis de résidus acides, est localisé dans la zone centrale de la région 1 et que le taux de phosphorylation pourrait moduler ses différentes activités régulatrices sur un promoteur de gène IE ou $\mathrm{E}$ [16]. Outre un signal de localisation nucléaire, situé dans la région 3 et similaire à ceux de l'ICP4 et de l'antigène $\mathrm{T}$ du virus SV40, l'IE62 présente, dans la fin de la région 1 ou le début de la région 2 , un domaine de distribution intranucléaire.

La protéine IE62 n'agit probablement pas seule mais en collaboration avec d'autres protéines IE. Les interactions existant entre l'IE62 et l'IE4 suscitent un intérêt croissant puisque la protéine IE4 augmente l'effet transactivateur de l'IE62 [15]. L'IE62 transporte la protéine IE4 du cytoplasme vers le noyau [22] et la région carboxy-terminale (région 4) de l'IE62 semble impliquée dans cette propriété. Néanmoins, l'interaction physique directe entre ces deux protéines n’a pas encore été démontrée. Cette étude prend tout son sens au vu des mécanismes d'action progressivement mis à jour pour les fonctions de l'IE4.

\section{La protéine IE4: un régulateur transcriptionnel et post-transcriptionnel}

L'IE4 appartient à une famille de protéines relativement bien conservées parmi les Herpesvirus telles l'ICP27 du HSV-1, le BMLF de l'EBV ou l'UL69 du CMV. De nombreuses informations ont notamment été accumulées sur les fonctions de la protéine ICP27 du HSV-1, ce qui a permis d'aiguiller les recherches sur l'IE4. L'ICP27 présente une grande similitude de séquence avec l'IE4, surtout dans la portion carboxy-terminale [3]. L'ICP27 déploie des propriétés de transactivation et de transrépression de l'expression des gènes du HSV-1 [23] et joue un rôle essentiel dans le cycle viral [24]. L'IE4 reste encore relativement peu caractérisée par rapport à l'ICP27. Bien que ses fonctions de transactivation de l'expression des gènes du VZV soient clairement établies, des inconnues subsistent quant aux mécanismes moléculaires impliqués dans ses propriétés. La protéine IE 4 (452 acides aminés, $52 \mathrm{kDa}$ ) (figure 4) joue probablement un rôle important dans le contrôle de l'expression des gènes au cours du cycle viral car elle est synthétisée très précocement au cours de l'infection productive (phase IE) [25] et se retrouve en grande quantité dans le tégument du virus [11]. En outre, des expériences de transfection transitoire de cellules eucaryotes indiquent que l'IE4 peut stimuler l'expression des gènes, soit seule, soit en synergie avec la protéine IE62 [15,18].

D'un point de vue mécanistique, l'ICP27 offre l'intérêt tout particulier de se comporter comme un régulateur post-transcriptionnel [26]. Il est vrai que la régulation du début de la transcription ne constitue que la première étape du processus menant à l'expression d'une protéine. L'étude de la régulation post-transcriptionnelle, relativement récente par rapport à celle de la régulation transcriptionnelle, propose un champ d'investigation passionnant. L'IE4 possède également des propriétés d'activateur post-transcriptionnel car les stimulations de certains promoteurs ne sont pas directement corrélées à des augmentations des quantités d'ARNm [18, 25]. Les mécanismes impliqués demeurent inconnus mais la région riche en arginine présente à l'extrémité amino-terminale de la molécule pourrait intervenir (figure 4). En effet, ces motifs également présents dans l'ICP27, ainsi qu'au sein d'autres protéines interagissant avec l'ARN, sont importants pour les fonctions régulatrices de cette protéine [27]. En outre, la construction de protéines chimériques entre l'ICP27 et l'IE4 a montré que les extrémités amino-terminales peuvent s'échanger entre les protéines sans altérer leurs fonctions. En revanche, leurs régions carboxyterminales ne sont pas interchangeables [28]. Ces extrémités riches en résidus cystéine sont cruciales pour leurs propriétés régulatrices [29]. 


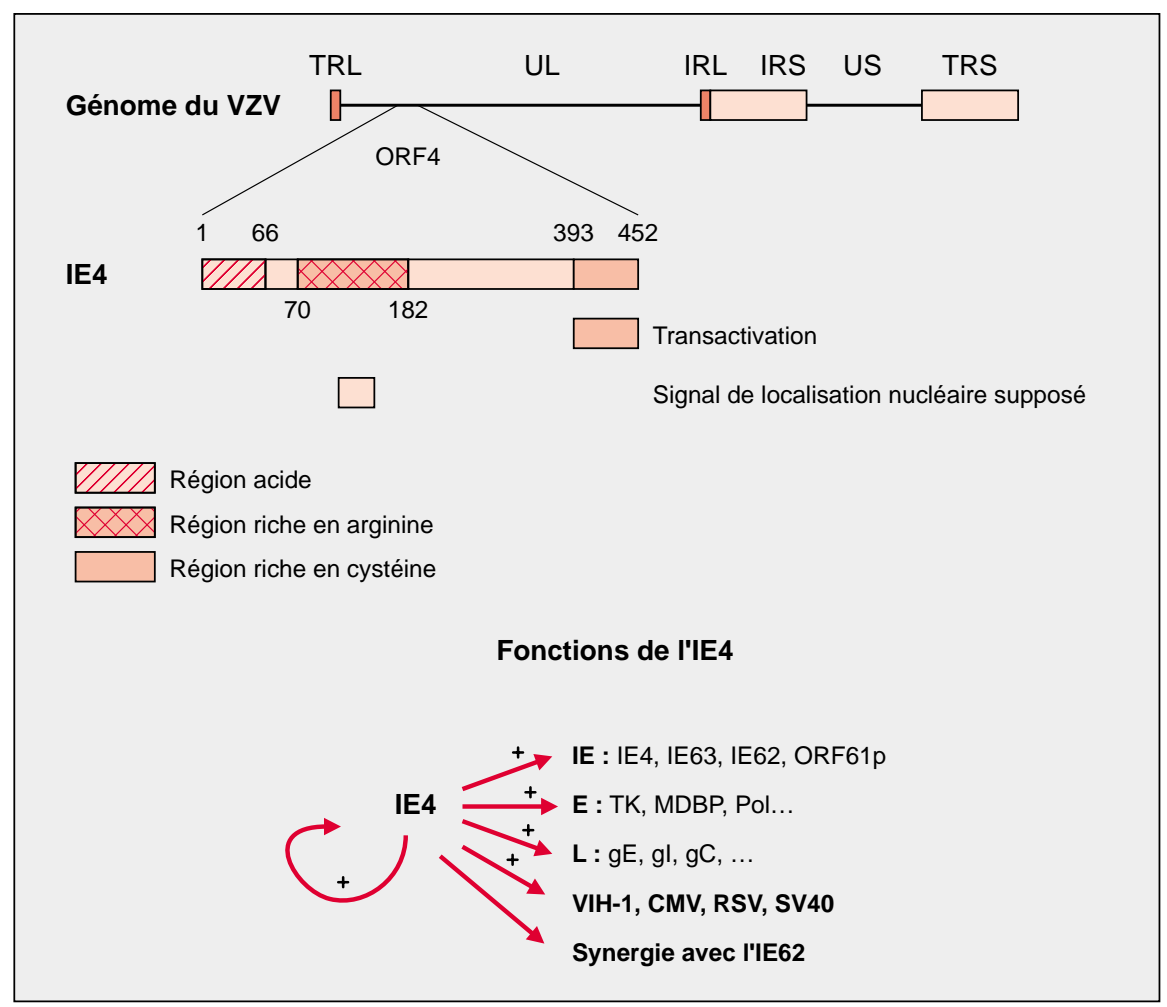

Figure 4. Fonctions de la protéine IE4 du VZV. L'ORF 4 du VZV code pour une protéine de 452 acides aminés nommée IE4. Ce facteur est capable de transactiver tous les promoteurs des gènes du VZV ainsi que des promoteurs hétérologues (VIH-1: virus de l'immunodéficience humaine-1; CMV: cytomegalovirus ; RSV: Rous sarcoma virus; SV40: simian virus 40). Elle agit en synergie avec I'IE62. Seule la région carboxy-terminale, riche en résidus cystéine et histidine, a été identifiée comme un domaine fonctionnel important pour la transactivation. Le rôle des régions acide et riche en résidus arginine est actuellement inconnu.

Une seconde hypothèse sur les fonctions de l'IE4 découle de sa capacité d'activation transcriptionnelle mise en évidence par l'équipe de Perera (NIAID, Bethesda, MD, USA) [29]. Ses études ont montré que l'IE4 fusionnée au domaine de liaison à l'ADN de la protéine GAL4 pouvait stimuler un promoteur minimal portant des sites de fixation pour cette protéine. La protéine IE4 seule n'est pas capable de transactiver un tel promoteur. Ces résultats suggèrent que la protéine portée au voisinage d'un promoteur est capable de stimuler son activité. Nos propres travaux confirment cette observation. L'IE4 semble interagir avec le complexe de transcription par l'intermédiaire de la TBP (TATA box binding protein) ou du TFIIB (transcription factor IIB) mais aussi avec des facteurs transcriptionnels comme le NF- $\mathrm{KB}$ régulation de l'expression de très nombreux gènes. Nous avons observé que la stimulation du LTR (long terminal repeat) du VIH-1 par l'IE4 requiert la présence du NF-кB. Une caractéristique étonnante de la protéine IE4 est sa localisation majoritairement cytoplasmique au sein des cellules infectées, fait unique parmi les membres de sa famille chez les Herpesvirus. Il est actuellement difficile de corréler cette observation avec ses fonctions. La seule donnée expérimentale dont nous disposons concerne la capacité de la protéine IE62 d'entraîner l'IE4 dans le noyau dans lequel cette dernière pourrait exercer une partie de ses fonctions [22]. Il se peut que cet effet soit lié aux propriétés de transactivation synergique de ces deux protéines. La localisation cytoplasmique peut également être associée aux propriétés de régulation post-transcriptionnelle.
La recherche en cours vise à identifier d'éventuels domaines fonctionnels dans l'IE4. Elle s'intéresse aussi aux interactions physiques directes entre l'IE4 et d'autres protéines cellulaires ou virales. Les nouveaux éléments qui en sortiront permettront de mieux comprendre le rôle de ce régulateur transcriptionnel et/ou post-transcriptionnel dans la réactivation du virus.

\section{IE63 et la latence}

Le dernier acteur intervenant dans le jeu subtil du contrôle de l'expression des gènes est la phosphoprotéine tégumentaire IE63 (45 kDa). L'IE63 est synthétisée de façon abondante dès les premières étapes du cycle productif du VZV. Elle se localise principalement dans le noyau, mais est également détectée dans le cytoplasme des cellules infectées [30]. La séquence de ses 278 acides aminés a peu de similitude avec celle de la protéine ICP22 du HSV-1. Les données de la littérature concernant les propriétés de l'IE63 ou de l'ICP22 sont rares. Néanmoins, l'étude des fonctions attribuées à l'IE63 a suscité un intérêt croissant après la découverte de l'abondance de ses transcrits dans les cellules de ganglions sensoriels infectés de façon latente par le VZV $[6,7]$.

Lors d'expériences d'expression transitoire, la protéine IE63 manifeste un faible effet répresseur sur l'expression de certains gènes de classe E (28 et 29) mais aucun effet transactivateur significatif. Cependant, en présence des autres protéines régulatrices du VZV, une répression des propriétés activatrices de l'IE4 a clairement pu être mise en évidence alors que les propriétés de l'IE62 ne semblent pas modifiées par l'IE63 [30]. Les mécanismes moléculaires impliqués dans les fonctions de l'IE63 demeurent inconnus à l'heure actuelle.

Le gène 63 est transcrit sous la forme de deux ARN polyadénylés de 1,3 et $1,9 \mathrm{~kb}$ [31] qui peuvent être le résultat de l'utilisation de plusieurs sites de début de la transcription localisés 88,153 et 243 pb en amont du codon ATG, initiateur de la traduction et de l'un ou l'autre des deux sites de terminaison potentiels. L'analyse de la région promotrice du gène 63 
montre également la présence de boîtes TATA potentielles situées 30 pb en amont des différents sites de début de transcription répertoriés. Bien que la présence de plusieurs sites dans la région promotrice soit encore inexpliquée, une hypothèse serait que, selon les conditions rencontrées, ces différents sites permettraient une expression à partir de l'une ou l'autre des régions promotrices. Chacun des promoteurs posséderait les signaux nécessaires au début de la transcription en fonction des différentes étapes de l'infection. Cette hypothèse prend encore plus d'intérêt en raison de la transcription de l'ORF63 tant durant le cycle productif qu'au cours de la période de latence du VZV.

Comme nous l'avons déjà signalé précédemment, la latence du VZV se différencie de celle des autres Alphaherpesvirus par l'absence de LAT et par la présence pendant la phase quiescente de transcrits correspondant à des protéines IE du cycle lytique. En raison de la concentration élevée d'ARN messagers correspondant au gène 63 dans les cellules de ganglions sensoriels infectés de façon latente par le VZV, la présence de la protéine IE63 a été recherchée dans ces cellules. Un modèle expérimental d'infection latente par le VZV résultant d'injections de cellules infectées au niveau des coussinets plantaires de la patte du rat a permis de la mettre en évidence, principalement dans le noyau et le cytoplasme des cellules neuronales des ganglions sensoriels [30]. Plusieurs axones situés à la base du nerf sciatique correspondant à ces ganglions se sont également révélés positifs. La protéine tardive $\mathrm{gE}$ n'a jamais pu être détectée dans les mêmes conditions ce qui confirme le caractère latent de l'infection. Ces données confirmées par l'analyse de ganglions sensoriels humains prélevés par autopsie à des niveaux ne présentant aucun signe de réactivation [32] constituent la toute première mise en évidence de la synthèse d'une protéine d'un Alphaherpesvirus au cours de la latence chez l'homme. La présence durant la période de latence du VZV de la protéine IE63, sous une forme identique ou non à celle observée lors de l'infection lytique, fait supposer que celle-ci pourrait jouer un rôle prépondérant dans l'établissement et/ou le maintien de la latence, voire même dans la réactivation du virus. L'ensemble des observations suggère l'existence d'un mécanisme de régulation de l'infection latente du VZV différent de ceux proposés pour les autres Alphaherpesvirus (figure 5). En effet, il était généralement admis qu'aucune protéine virale de ces virus n'était synthétisée durant la latence, principalement caractérisée par la présence de LAT. Par conséquent, la mise en évidence à ce moment d'une protéine qui est, en outre, également synthétisée durant la phase IE du cycle productif, démontre une nouvelle fois le caractère particulier du VZV au sein de cette famille. Des études récentes conduisent à penser que l'IE4 et l'IE62 pourraient également être présentes pendant la latence [Gershon et al., communication personnelle]. La protéine IE63 et, éventuellement, l'IE62 et l'IE4, synthétisées tant au cours de la phase précoce immédiate de l'infection productive que pendant la période de latence, pourraient donc jouer un rôle essentiel dans le contrôle de la synthèse en cascade des protéines du virus.
Par ailleurs, la protéine IE63 constitue, au même titre que d'autres protéines structurales telles que l'IE62 ou la gpII, une cible importante du système immunitaire, suscitant une réponse immune complète. En effet, des tests de prolifération lymphocytaires ont permis de montrer que $59 \%$ des individus adultes sains, ayant précédemment développé la varicelle et dont les lymphocytes $\mathrm{T}$ prolifèrent en présence d'antigènes viraux non fractionnés, possédent des cellules T-mémoire reconnaissant la protéine IE63 purifiée [33]. En outre, la réponse T-cytotoxique vis-à-vis de l'IE63 est du même ordre que celle suscitée par l'IE62, dont le caractère immunogène a été caractérisé [34]. La détection, dans le sérum de ces donneurs, d'IgG reconnaissant spécifiquement la protéine IE63 ainsi que la mise en évidence d'une réponse immunitaire cellulaire complète confirment le caractère immunogène de la protéine IE63. Compte tenu des observations cliniques suggèrant une corrélation entre la fréquence de réactivations virales et le statut immunitaire, la protéine IE63 pourrait non seulement jouer un rôle-clé dans

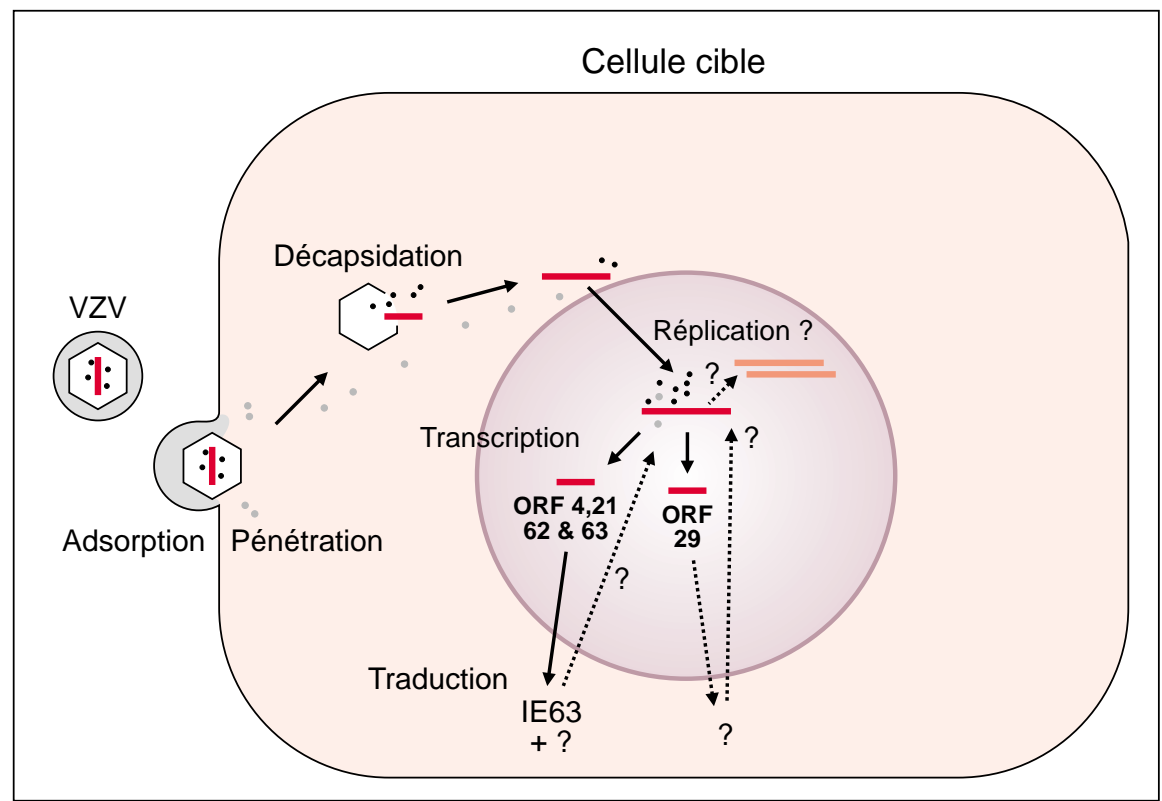

Figure 5. Cycle infectieux latent du VZV. Lors de l'infection latente de cellules nerveuses par le VZV, il y aurait une dérégulation de l'expression séquentielle des gènes du virus qui conduirait à la transcription restreinte de quelques $\operatorname{ORF}(4,62,63,21$ et 29) et à la traduction de la protéine IE63. Les protéines correspondant aux autres ORF (IE4, IE62, ORF21p et la major DNA binding protein codée par l'ORF 29) n'ont pas encore été mises en évidence de manière certaine. 
l'établissement et/ou le maintien de la latence, mais également assurer un contrôle de cet état latent par le système immunitaire. Une meilleure caractérisation de la réponse immune spécifiquement dirigée contre une ou des protéines synthétisées pendant la phase de quiescence permettrait certainement de clarifier l'importance du système immunitaire cellulaire.

\section{Conclusions}

La régulation de l'expression des gènes est cruciale pour le bon déroulement du cycle réplicatif du VZV dans la cellule infectée. Or, les processus d'établissement, de maintien et de réactivation de la latence sont probablement liés à la nature particulière de l'environnement neuronal qui dérègle l'expression des gènes et empêche l'accomplissement du cycle lytique. A l'heure actuelle, les mécanismes sous-tendant ces différents processus sont encore loin d'être connus en raison des difficultés de produire du virus in vitro et de l'absence de modèle animal reproduisant à la fois les phases d'infection primaire, de latence et de réactivation. Pourtant, nonobstant ces obstacles conséquents, certains ARN messagers codant pour des protéines régulatrices (IE62, IE 4 et IE63 notamment) ont pu être détectés pendant la latence. Cette particularité distingue le VZV des autres membres de la sous-famille des Alphaherpesvirus. Le contrôle des premières étapes du cycle est primordial pour l'orientation vers une infection lytique ou latente et les protéines virales les plus susceptibles d'intervenir dans la régulation de ces étapes initiales doivent, soit être apportées par le virus, soit être synthétisées durant les phases très précoces du cycle. Par conséquent, les études discutées ici soulignent l'importance des protéines IE4, IE62 et IE63 dans le contrôle séquentiel de l'expression des gènes du VZV et de ses cycles infectieux. Comme la latence du VZV résulte probablement d'une dérégulation de l'expression des gènes viraux à la suite des modifications quantitatives ou qualitatives des protéines présentes dans les cellules (neuronales ou gliales) infec- ment comprendre l'intérêt d'étudier les aspects fondamentaux de la régulation des gènes du VZV. Leur connaissance devrait, dans l'avenir, conduire au développement de nouvelles stratégies antivirales permettant de mieux contrôler les maladies qui sont associées à l'infection par le VZV

\section{RÉFÉRENCES}

1. Weller TH. Serial propagation in vitro of agents producing inclusion bodies derived from varicella and herpes zoster. Proc Soc Exp Biol Med 1953; 83 : 340-6.

2. Ostrove JM. Molecular biology of varicella zoster virus. Adv Virus Res 1990; 38: 4598 .

3. Davison AJ, Scott JE. The complete DNA sequence of varicella-zoster virus. J Gen Virol $1986 ; 67$ : 1759-816.

4. Gilden DH, Mahalingam RN, Dueland N, Cohrs R. Herpes zoster: pathogenesis and latency. In: Melnick JL, ed. Progr Med Virol. Basel, Karger, 1992; 39: 19-75.

5. Enquist LW. Infection of the mammalian nervous system by pseudorabies virus (PRV). Semin Virol 1994; 5 : 221-31.

6. Vafai A, Murray RS, Wellish M, Devlin M, Gilden DH. Expression of varicella-zoster virus in normal human trigeminal ganglia. Proc Natl Acad Sci USA 1988; 85 : 2362-6.

7. Sadzot-Delvaux C, Merville-Louis MP, Delree P, Marc P, Piette I, Moonen G, Rentier $\mathrm{B}$. In vivo model for varicella-zoster virus persistent infection of dorsal root ganglia. J Neurosci Res 1990; 26: 83-9

8. Cohrs R, Mahalingam R, Dueland A, Wolf W, Wellish M, Gilden D. Restricted transcription of VZV in latently-infected human trigeminal and thoracic ganglia. J Infect Dis 1992; 166: S24-S29.

9. Kinchington PR, Hougland JK, Arvin AM, Ruyechan WT, Hay J. The varicella-zoster virus immediate-early protein IE62 is a major component of virus particles. $J$ Virol 1992; 66: 359-66.

10. Moriuchi H, Moriuchi M, Straus SE, Cohen JI. Varicella-zoster virus open reading frame 10 protein, the herpes simplex virus VP16 homolog, transactivates herpesvirus immediate-early gene promoters. J Virol $1993 ; 67: 2739-46$.

11. Kinchington PR, Bookey D, Turse SE. The transcriptional regulatory proteins encoded by varicella-zoster open reading frames (ORFs) 4 and 63, but not ORF61, are associated with purified virus particles. J Virol 1995; 69: 4274-82.

12. Cohen JI, Seidel, KE. Varicella-zoster virus (VZV) open reading frame 10 , the homolog of the essential herpes simplex virus protein VP16, is dispensable for replication in vitro. Proc Natl Acad Sci USA 1993; 90: 7376-80.

13. Leib D, Coen D, Bogard C, Hicks K, Yager D, Knipe D, Tyler K, Schaffer P. Immediate-early regulatory gene mutants define different stage in the establishment and reactivation of herpes simplex virus latency. J Virol 1989; 63: 759-68.

14. Disney GH, Everett RD. A herpes simplex type 1 recombinant with both copies of the Vmw175 coding sequences replaced by the homologous varicella-zoster virus open reading frame. J Gen Virol 1990; 71 : 897-906.

15. Perera LP, Mosca JD, Ruyechan WT, Hay J. Regulation of varicella-zoster virus gene expression in human T-lymphocytes. jVirol 1992; 66: 5298-304.

16. Baudoux L, Defechereux P, Schoonbroodt S, Merville MP, Rentier B, Piette J. Mutational analysis of varicella-zoster virus major immediate-early protein IE62. Nucleic Acids Res 1995; 23 : 1341-9.

17. Moriuchi M, Moriuchi H, Straus SE, Cohen JI. Varicella-zoster virus (VZV) virionassociated transactivator open reading frame 62 protein enhances the infectivity of VZV DNA. Virology 1994; 200 : 297-300.

18. Defechereux P, Melen L, Baudoux L, Merville-Louis MP, Rentier B, Piette J. Characterization of the regulatory functions of varicella-zoster virus open reading frame 4 gene product. J Virol 1993; 67: 4379-85.

19. Tyler JK, Everett RD. The DNA binding domains of the varicella-zoster virus gene 62 and herpes simplex type 1 ICP 4 transactivator proteins heterodimerize and bind to DNA. Nucleic Acids Res 1994; 22: 711-21.

20. Tyler JK, Everett RD. The DNA binding domain of the varicella-zoster virus gene 62 protein interacts with multiple sequences which are similar to the binding site of the related protein of herpes simplex virus type 1. Nucleic Acids Res 1993; 21: 513-22.

21. Perera LP, Mosca JD, Ruyechan WT, Hayward GS, Straus SE, Hay J. A major transactivator of varicella-zoster virus, the immediate-early protein IE62, contains a potent N-terminal activation domain. J Virol 1993; 67 : 4474-83.

22. Defechereux P, Debrus S, Baudoux L, Schoonbroodt S, Merville MP, Rentier B, Piette J. Intracellular distribution of the ORF4 gene product of varicella-zoster virus is influenced by the IE62 protein. J GenVirol 1996; 77 : 1505-13.

23. Rice SA, Su L, Knipe DM. Herpes simplex virus a protein ICP27 possesses separable positive and negative regulatory activities. JVirol 1989; 63: 3399-407.

24. McCarthy A, McMahan L, Schaffer PA. Herpes simplex virus type 1 ICP27 deletion exhibits altered pattern of transcription and are DNA deficient. J Virol 1989; 63: 18-27.

25. Defechereux P, Debrus S, Baudoux L, Rentier B, Piette J. Varicella-zoster virus open reading frame 4 encodes an immediate-early protein with post-transcriptional regulatory properties. J Virol 1997; 71: 7073-9. 


\section{RÉFÉRENCES}

26. Sandri-Goldin RM, Mendoza GE. A herpes virus regulatory protein appears to act post-transcriptionally by affecting mRNA processing. Genes Dev 1992; 6: 848-63.

27. Hibbard MK, Sandri-Goldin RM. Arginine-rich regions succeeding the nuclear localization region of the herpes simplex virus type 1 regulatory protein ICP27 are required for efficient nuclear localization and late gene expression. J Virol 1995; 69 : 4656-67.

28. Moriuchi M, Moriuchi H, Debrus S, Piette J, Cohen JI. The acidic amino-terminal domain of varicella-zoster virus open reading frame 4 protein is required for transactivation and can functionally replace the corresponding domain of herpes simplex virus ICP27. Virology 1995; 208: 376-82.

29. Perera LP, Kaushal S, Kinchington PR, Mosca JD, Hayward GS, Straus SE. Varicellazoster virus open reading frame 4 encodes a transcriptional activator that is functionally distinct from that of herpes simplex virus homolog ICP27. J Virol 1994; 68: 2468-77.

30. Debrus S, Sadzot-Delvaux C, Nikkels AF, Piette J, Rentier B. Varicella-zoster virus gene 63 encodes an immediate-early protein abundantly expressed during latency. J Virol 1995; 69: 3240-5.

31. Kinchington PR, Vergnes JP, Defechereux $\mathrm{P}$, Piette $\mathrm{J}$, Turse SE Transcriptional mapping of the varicella-zoster virus regulatory genes encoding open reading frames 4 and 63. I Virol 1994; 68: 3570-81.

32. Mahalingam R, Wellish M, Cohrs R, Debrus S, Piette I, Rentier B, Gilden DH. Expression of protein encoded by varicellazoster virus open reading frame 63 in latently infected human ganglionic neurons. Proc Natl Acad Sci USA 1996; 93: 2122-4.

33. Sadzot-Delvaux C, Kinchington PR, Debrus S, Rentier B, Arvin AM. Recognition of the latency-associated immediate early protein IE63 of varicella-zoster virus by human memory T-lymphocytes. I Immunol 1997 ; 159 : 2802-6.

34. Arvin AM, Sharp M, Smith S, Koropchak CM, Diaz PS, Kinchington PR, Ruyechan W, Hay J. Equivalent recognition of a varicellazoster virus immediate early protein (IE62) and glycoprotein I by cytotoxic T-lymphocytes of either CD4+ or CD8+ phenotype. J Immunol 1991 ; 146 : 257-65.

TIRÉS À PART

J. Piette

$\mathrm{m} / \mathrm{s}^{\circ} 5$, vol. 14 , mai 98

\section{Summary}

Regulation of the infectious cycles of the varicella-zoster virus

Varicella-zoster virus (VZV) is an Alphaherpesvirus responsible for two human diseases : primary exposure to the virus results in chicken pox (varicella) and reactivation following a period of latency in dorsal root ganglia gives rise to shingles (zoster). Interestingly, several transcripts corresponding to regulatory proteins present during the lytic cycle can be found in latently infected cells. The IE62 protein, component of the viral tegument, is a nuclear phosphoprotein. IE62 may play a crucial role in triggering and regulating the replicative cycle of VZV since it transactivates all classes of VZV genes and is able to repress or activate its own promoter. Moreover, IE62 acts in synergy with IE4, another important regulatory protein, to stimulate VZV gene promoters and IE62 is responsible for the translocation of IE4 from the cytoplasm to the nucleus. IE4 is expressed at very early times of the VZV productive cycle. Predominantly localized in the cytoplasm, IE4 activates several VZV genes, either alone or in synergy with IE62, as well as heterologous viral genes. At the molecular level, IE4 seems to act both transcriptionally and post-transcriptionally. Another major VZV protein is a $45 \mathrm{kDa}$ phosphorylated protein, called IE63, which is abundantly expressed at the onset of the productive cycle. It is also detected during latency in humans and in a rat animal model, an unexpected observation in Alphaherpesviruses. IE63 displays little direct effect on VZV gene promoters, it shows no inhibitory effect on the transactivating functions of IE62 but it represses the IE4mediated activation. Studies conducted to define the mode of action of three VZV regulatory proteins playing crucial roles in the latency and reactivation of the virus will not only lead to a better understanding of the virus pathogenesis but will probably help define novel therapeutic tools.
Société Française de Biochimie et Biologie Moléculaire Colloque du groupe thématique Phosphorylation des protéines Arcachon

\section{1-23 septembre 1998}

Le colloque couvrira les différents aspects de l'étude des protéinekinases et des phosphoprotéines phosphatases.

\section{THĖMES ABORDÉS}

\begin{tabular}{l|l}
$\square$ Signalisation & $\begin{array}{c}\text { Interactions } \\
\text { cellulaires }\end{array}$ \\
$\square$ Différenciation & $\begin{array}{l}\text { Trafic } \\
\text { intracellulaire }\end{array}$ \\
$\square$ Prolifération & $\begin{array}{l}\text { Études } \\
\text { structurales }\end{array}$ \\
$\square$ Dynamique & $\square$ Etc. \\
cellulaire &
\end{tabular}

Les présentations auront lieu sous la forme de communications orales brèves ou d'affiches. La participation de jeunes chercheurs est vivement encouragée.

\section{INSCRIPTIONS ET INFORMATIONS}

La date limite de préinscription est le 15 janvier 1998. Renseignements auprès du Pr Bernard Ducommun IPBS - Cnrs,

205, route de Narbonne

31077 Toulouse Cedex, France

Tél. : 0561175931

Fax : 0561175905 sfbbm98@ipbs.fr

L'accès à Arcachon est simple : gare SNCF-TGV

à proximité du palais des congrès, aéroport de Bordeaux-Mérignac à $30 \mathrm{mn}$.

COMITÉ D'ORGANISATION

Pr Bernard DUCOMMUN (Toulouse), Dr Michèle CAIZERGUES-FERRER (Toulouse)

Dr Michel VERON (Paris) 